\title{
Detector de IR de lámina ferroeléctrica de $(\mathrm{Pb}, \mathrm{Ca}) \mathrm{TiO}_{3}$
}

\author{
P. RAMOS*, J. MENDIOLA, R. JIMENEZ, M.L.CALZADA, A.GONZÁLEZ, P. TEJEDOR \\ *Dpto de Electrónica, Universidad de Alcalá, Alcalá de Henares, E-28871 Madrid \\ Inst. Ciencia de Materiales de Madrid (C.S.I.C.) Cantoblanco, E-28049 Madrid
}

\begin{abstract}
Se han obtenido depósitos multicapa de titanato de plomo modificado con calcio, $\left(\mathrm{Pb}_{0.76} \mathrm{Ca}_{0.24}\right) \mathrm{TiO}_{3^{3}}$ mediante un método de sol-gel, sobre substratos de $\mathrm{Pt} / \mathrm{MgO}(100)$. El tratamiento térmico del substrato y la cristalización de las multicapas mediante tasas de calentamiento rápidas causan el desarrollo de una importante orientación preferente según el eje polar, $<001>$ perpendicular al mismo, lo que supone una autopolarización muy rentable para su empleo en detectores piroeléctricos de radiación infrarroja. Mediante una configuración de electrodos discretos se fabrican minicondensadores de $7.10^{-3} \mathrm{~cm}^{2}$ de área con los que se caracteriza ópticamente el detector para condiciones estándar: cuerpo negro a $500 \mathrm{~K}$, modulación mecánica de la radiación entre $1-20 \mathrm{~Hz}$, una electrónica de acondicionamiento de la señal de respuesta y un amplificador sintonizado para medir la respuesta en voltaje. Se analiza el efecto de los tres factores que intervienen en la fabricación del detector: a) características del material piroeléctrico (figuras de mérito del material), b) ensamblado de las partes del detector (substrato, electrodos, conexiones, etc.) y c) diseño del circuito electrónico de medida (etapa de preamplificación, de filtrado y de amplificación), resaltando la necesidad del aislamiento térmico.
\end{abstract}

Palabras clave: Detector IR, ferroeléctrico y titanato de plomo modificado.

\section{IR Detectors made of ferroelectric $(\mathrm{Pb}, \mathrm{Ca}) \mathrm{TiO}_{3}$ thin film}

A sol-gel $\left(\mathrm{Pb}_{076} \mathrm{Ca}_{024}\right) \mathrm{TiO}_{3}$ solution was deposited onto $\mathrm{Pt} / \mathrm{MgO}(100)$ substrates. Previous thermal treatment of the substrate and the high rate of crystallization heating promote an important preferred orientation along the polar axis, $<001>$ and therefore selfpolarization, very convenient for the use of IR pyroelectric detectors. By depositing circular electrodes, $7.10^{-3}$ $\mathrm{cm}^{2}$ of area, an array of small capacitors are developed which are characterized as detectors in standard conditions: radiation from a black-body at $500 \mathrm{~K}$, modulated between $1-20 \mathrm{~Hz}$, a lock-in amplifier and an electronic circuit to sense and treat the electrical response. Three main factors affecting the detector performances are analyzed: a) Figures of merit of the pyroelectric material; b) assembly of the whole parts of detector (substrate, electrodes, leads, frame, etc) and c) electronic circuitry to sense and amplify signals. Thermal isolation is concluding as the most important fact to improve responsivity.

Key words: IR Detector, ferroelectric and modified lead titanate.

\section{INTRODUCCCIÓN}

Una de las aplicaciones más atractivas de los materiales ferroeléctricos es la fabricación de detectores de radiación infrarroja, IR, empleando la propiedad piroeléctrica, que permite convertir en señal eléctrica la variación de la polarización eléctrica $\mathrm{P}$ del material al cambiar su temperatura un $\Delta \mathrm{T}$, de acuerdo con la expresión $\mathrm{I}=\mathrm{S}^{*}(\mathrm{dP} / \mathrm{d} \Delta \mathrm{T})$, siendo $\mathrm{S}$ es la superficie sensible. Con tales materiales se pueden construir detectores altamente competitivos como los dispositivos fotónicos o cuánticos de naturaleza semiconductora, que aunque muy sensibles requieren ser enfriados hasta temperaturas del nitrógeno líquido para reducir el ruido térmico, lo que además de elevar su precio reduce su empleo a longitudes de ondas $\lambda<20 \mu \mathrm{m}$. Desde la década de los setenta hasta ahora se ha producido una gran evolución en la fabricación de dispositivos piroeléctricos tanto puntuales como formadores de imágenes térmicas, habiendo pasado sus aplicaciones del campo estrictamente militar al civil. Ello ha sido debido al desarrollo de las técnicas de preparación de materiales y a la tecnología de circuitos integrados que ha permitido pasar del empleo de monocristales o cerámicas en forma masiva, a láminas delgadas en circuitos integrados de silicio [1].

En la fabricación de dispositivos piroeléctricos tanto para la detección de radiación infrarroja puntual como en la ter- mografia, se siguen contemplando tres factores a tener en cuenta para su optimización: a) las propiedades del material que vienen dadas por sus figuras de mérito; b) el ensamblaje del material en forma de lámina depositada sobre un substrato y con los necesarios electrodos y pistas para extraer la señal eléctrica; tal vez sea el factor que entrañe mayor dificultad tecnología, ya que se trata de aumentar el rendimiento del elemento sensor haciendo que la mayor parte de la energía térmica que le llegue sea trasformada en señal eléctrica medible. Se persigue optimizar el circuito térmico para reducir pérdidas de calor, lo que exige técnicas de micro-mecanización y depósitos de capas de elevada absorción; y c) circuitos electrónicos de altas prestaciones que permitan una elevada amplificación y bajo ruido.

En el presente trabajo se aborda la viabilidad de un detector piroeléctrico de IR aprovechando las posibilidades de la técnica de sol-gel de depositar láminas de titanato de plomo modificado con calcio, que presentan un importante coeficiente piroeléctrico espontáneo, debido a la elección de los substratos y los tratamientos térmicos aplicados tanto a los substratos como a las láminas. Se analiza la vía de reducir las pérdidas térmicas en el substrato y la selección de los componentes eléctricos 


\section{PROCEDIMIENTO EXPERIMENTAL.}

\subsection{Preparación y ensamblaje del detector piroeléctrico.}

Se preparan mediante la técnica sol-gel soluciones precursoras de $\left(\mathrm{Pb}_{0.76} \mathrm{Ca}_{0.24}\right) \mathrm{TiO}_{2}$ con una concentración molar $\sim 0.3$ con un $10 \%$ de $\mathrm{PbO}$ en exceso. El substrato sobre el que se hace el deposito es $\mathrm{Pt} / \mathrm{MgO}(100)$; el platino se ha depositado mediante pulverización catódica empleando una máscara de modo que se forman electrodos de forma circular de $1 \mathrm{~mm}$ de diámetro con una disposición matricial de 2 columnas por 3 filas para su posterior caracterización piroeléctrica (figura 1). El tamaño de los substratos empleados es de $10^{*} 10^{*} 0.5 \mathrm{~mm}^{3} \mathrm{y}$ $10^{*} 10^{*} 0.2 \mathrm{~mm}^{3}$, recocidos previamente a $800{ }^{\circ} \mathrm{C}$. Las láminas delgadas se forman mediante sucesivas etapas de depósito por centrifugación y cristalización de dos capas a $700{ }^{\circ} \mathrm{C}$ durante $50 \mathrm{~s}$, calentadas a un ritmo de $30^{\circ} \mathrm{C} / \mathrm{s}$ [2]. Así se consiguen láminas de aproximadamente $300 \mathrm{~nm}$ de espesor, de fase única perowskita y con una fuerte orientación preferente según el eje polar $<001>$ que resulta en un notable coeficiente piroeléctrico neto. Sobre las láminas se han depositado electrodos de Ni-Cr empleando las mismas máscaras que para los electrodos inferiores, con lo que se consiguen seis minicondensadores con electrodos inferiores conectados a uno común y superiores discretos con alta emisividad que se emplean para las medidas de caracterización y posterior ensamblaje en el dispositivo del detector de infrarrojo. Antes de su ensamblaje final se ha realizado un ataque químico controlado de la parte inferior del substrato, empleando una solución de ácido fosfórico. El ataque solamente se ha producido debajo de cada minicondensador, fabricando pozos para aumentar la respuesta del detector. La medida del efecto del ataque se hizo por técnicas de perfilometría. Las conexiones finales de los minicondensadores fueron realizadas por micro-soldadura de hilo de $\mathrm{Al}$ a un zócalo (figura 1) diseñado para conectarlo a la electrónica de acondicionamiento.

\subsection{Caracterización del detector piroeléctrico.}

Las medidas del coeficiente piroeléctrico, $\gamma$ se han realizado sometiendo a las laminas a una onda térmica triangular y midiendo la corriente generada de origen piroeléctrico que describe una onda cuadrada [3]. El grado de orientación preferente de las láminas fue evaluado mediante difracción de Rayos X utilizando la geometría Bragg-Brentano en un difractómetro Siemmes D-500 [4]. La constante dieléctrica , $\varepsilon_{\mathrm{R}}$ y la tangente de pérdidas dieléctricas, tan $\delta$ se han medido empleando un puente de impedancias, HP-4284 A a una frecuencia de un $1 \mathrm{kHz}$ y un voltaje de $100 \mathrm{mV}$.

La caracterización óptica del detector piroeléctrico se ha realizado por un método dinámico (figura 2), que consiste en utilizar un cuerpo negro a $500 \mathrm{~K}$ como fuente de radiación infrarroja, modulada por un chopper mecánico a varias frecuencias (1-20 Hz). La electrónica de acondicionamiento unipolar optimizada (figura 3a) está compuesta de tres partes básicas: un adaptador de impedancias necesario para convertir en voltaje la señal de respuesta en corriente, un preamplificador y una etapa de amplificación diferencial con un filtro paso banda centrado en $7 \mathrm{~Hz}$ con un ancho de banda de $1.14 \mathrm{~Hz}$ con ruidos electrónicos mínimos.

La electrónica de acondicionamiento y el detector piroeléctrico se encuentran conectados y apantallados en un caja metálica con un filtro de IR de silicio (con una atenuación $\sim 80 \%$

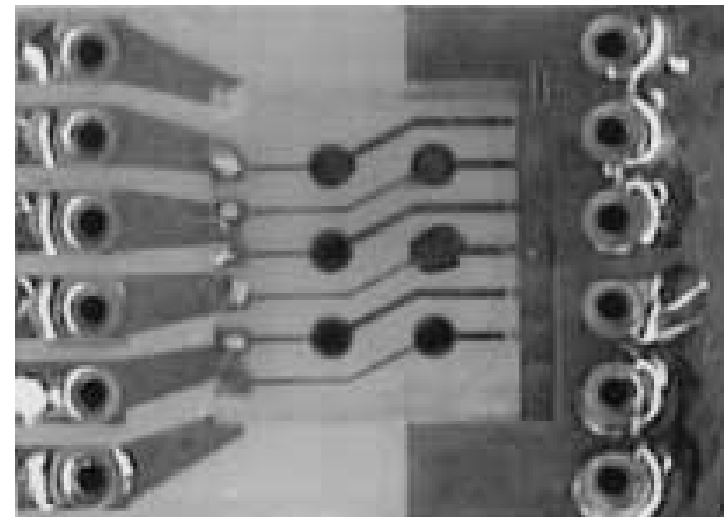

Figura 1. Fotografía de los minicondensadores piroeléctricos crecidos sobre $\mathrm{MgO}$ y sus microsoldaduras al zócalo.

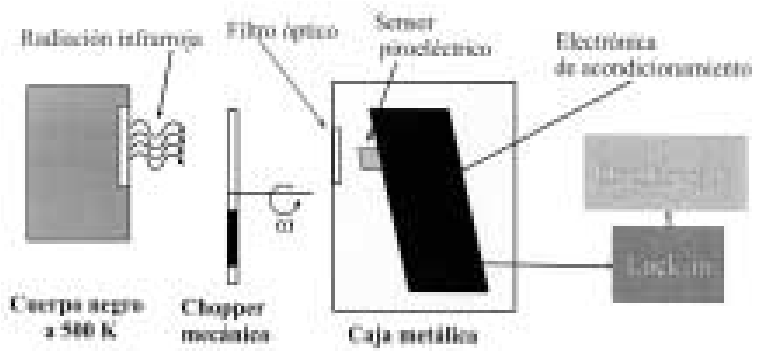

Figura 2. Esquema del sistema de caracterización óptica del detector piroeléctrico.

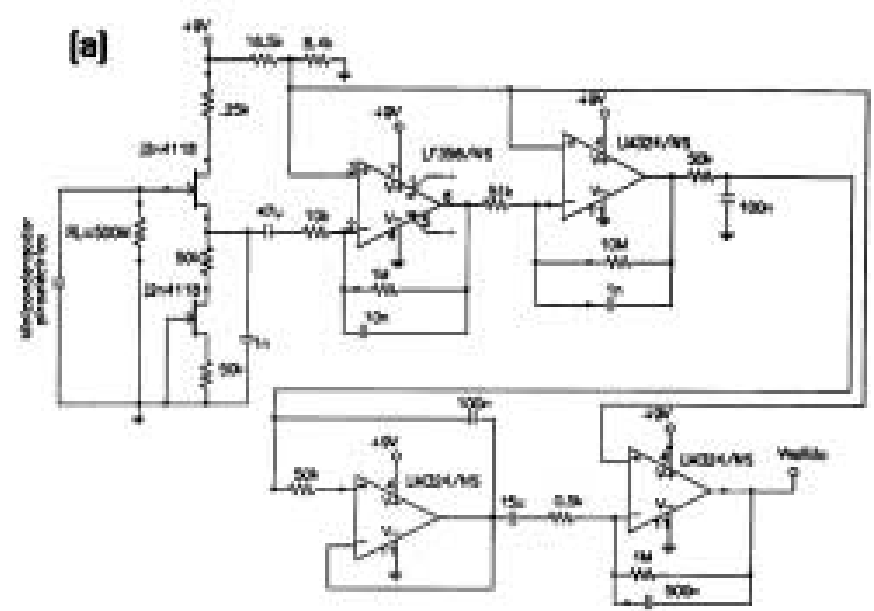

[b]
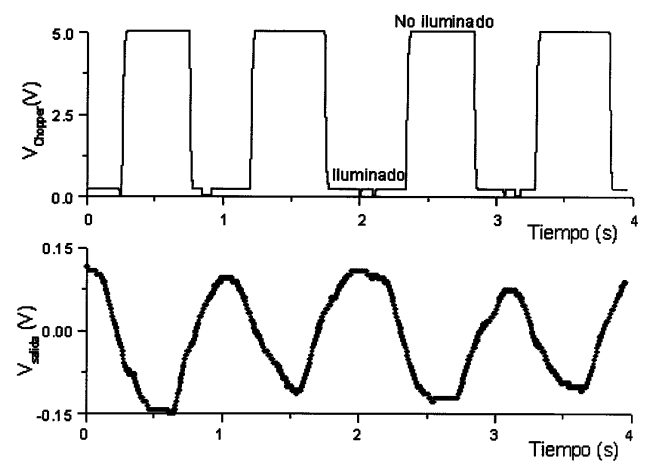

Figura 3. a) Electrónica de acondicionamiento y b) evolución temporal de la señal de salida, $\mathrm{V}_{\text {salida }}$ del detector para una frecuencia de modulación de $1 \mathrm{~Hz}$. 
entre longitudes de onda de 2 a $25 \mu \mathrm{m}$ ) centrado en la superficie sensible del detector. El ruido y el voltaje de salida de la electrónica se han medido con un "Lock-in amplifier" y un osciloscopio. En la figura $3 \mathrm{~b}$ se muestra la respuesta típica del detector.

\section{RESULTADOS Y DISCUSIÓN.}

En el difractograma de las láminas de $\left(\mathrm{Pb}_{0.76} \mathrm{Ca}_{0.24}\right) \mathrm{TiO}_{3}$ depositadas sobre $\mathrm{Pt} / \mathrm{MgO}(100)$ con un electrodo de Pt extenso (figura 4) se observa una orientación preferente en la dirección $<001>$, evaluada como $\alpha_{c}=0.87$ de acuerdo con Kim et al.[5]. Esto justifica que la lámina muestre un coeficiente piroeléctrico apreciable sin necesidad de polarización que además no se degrada con el tiempo (figura 5), particularidad de especial interés en la elección de dicho material.

La caracterización dieléctrica y piroeléctrica previa para evaluar los minicondensadores se detallan en la tabla I. Con el propósito de evaluar las láminas obtenidas para un detector de IR, se calcularon las figuras de mérito del material a partir de las propiedades medidas en los minicondensadores.

$$
F_{V}=\frac{\gamma}{C^{E} \varepsilon_{R} \varepsilon_{o}} \quad \text { y } \quad F_{D}=\frac{\gamma}{\sqrt{C^{E} \varepsilon_{R} \varepsilon_{o} \tan \delta}}
$$

donde $\varepsilon_{\mathrm{o}}$ es la constate dieléctrica en el vacío $\left(=8.8510^{-14} \mathrm{~F} / \mathrm{cm}\right)$ y $\mathrm{C}^{\mathrm{E}}$ es el calor especifico del material $\left(\sim 2.5 \mathrm{~J} \mathrm{~cm}^{-3} \mathrm{~K}^{-1}\right)$. Si bien tales características no bastan para obtener las prestaciones del detector, puesto que dependen de dos factores: la conexión de los minicondensadores con los dispositivos de medida (electrónica de acondicionamiento) y el paso de la energía térmica para su conversión en señal eléctrica medible (circuito térmico). Como en cualquier proceso de detección térmica -en el que presente, la conversión de un incremento de temperatura $\Delta \mathrm{T}$ en carga eléctrica liberada, I medida sobre una resistencia de carga $R_{L}$ (figura 3a)-, lo importante es que el detector absorba la mayor cantidad de calor posible, provocando un aumento de su respuesta que se optimiza reduciendo las pérdidas térmicas por conducción térmica a través del entorno: en este caso el substrato que soporta a la lámina.

Planteando la ecuación de transmisión de calor,

$$
H \frac{d \Delta T}{d t}+G \Delta T=\eta P(t)
$$

donde la potencia de radiación está modulada por el chopper $\left(\mathrm{P}(\mathrm{t})=\mathrm{P}_{0} \mathrm{e}^{\mathrm{jwt}}\right)$, siendo $\mathrm{f}=\omega / 2 \pi$ la frecuencia de modulación, $\eta$ la emisividad del electrodo superior, $\mathrm{H}$ la capacidad calorífica del elemento sensor, expresada en $(\mathrm{J} / \mathrm{K})$ y $\mathrm{G}$ su conductancia térmica con el entorno (substrato), expresada en $\mathrm{W} / \mathrm{K}$, su solución es de la forma:

$$
\Delta \mathrm{T}=\frac{\eta \mathrm{P}_{\mathrm{O}}}{\mathrm{G}\left(1+\omega^{2} \tau_{\mathrm{t}}^{2}\right)^{1 / 2}}
$$

donde $\tau_{t}$ es la constante de tiempo térmico e igual a $H / G$, que toma la forma de:

$$
\Delta T=\frac{\eta P_{O}}{G} \quad \text { (para baja frecuencia) } \text { y } \Delta T=\frac{\eta P_{O}}{G \omega \tau_{t}} \text { (para alta frecuencia) } \text { (4) }
$$

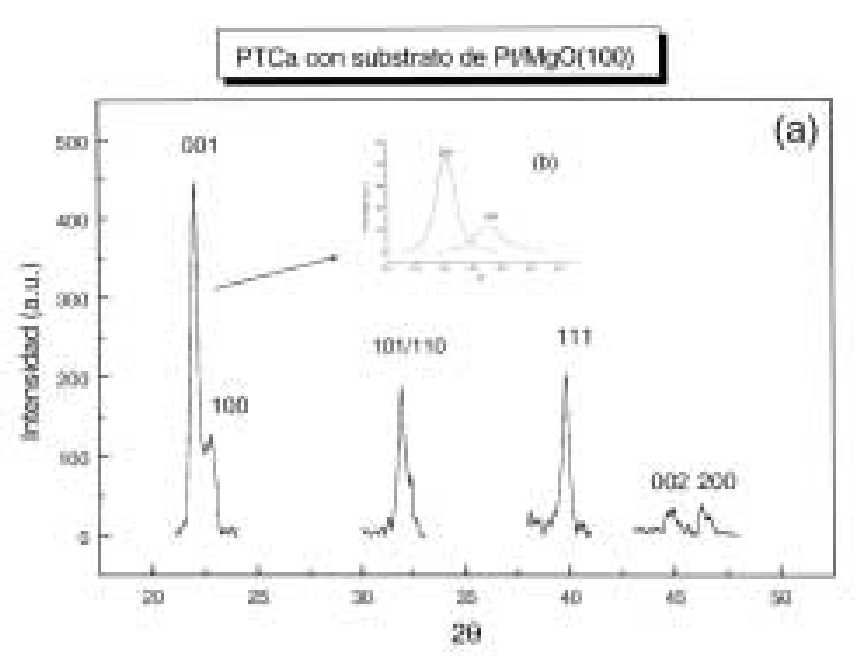

Figura 4. a) Difractograma de láminas delgadas de $\left(\mathrm{Pb}_{0.76} \mathrm{Ca}_{0.24}\right) \mathrm{TiO}_{3}$ depositadas sobre $\mathrm{Pt} / \mathrm{MgO}(100)$. b) Doblete de los picos de difracción 001/100 resultantes de la deconvolución, para visualizar la orientación preferente.

TABLA I. PROPIEDADES DIELÉCtRICAS Y PIROELÉCtRICAS DEL DETECTOR PIROELÉCTRICO

\begin{tabular}{|c|c|c|c|c|c|c|}
\hline $\begin{array}{c}\text { Tamaños de los } \\
\text { substrato de } \\
\mathrm{MgO}\end{array}$ & $\begin{array}{c}\varepsilon_{\mathrm{R}} \\
\text { a } 1 \mathrm{kHz}\end{array}$ & $\begin{array}{c}\tan \delta \\
\text { a } 1 \mathrm{kHz}\end{array}$ & $\begin{array}{c}\mathrm{C}^{\mathrm{E}} \\
\left(\mathrm{J} \mathrm{cm}^{-3} \mathrm{~K}^{-1}\right)\end{array}$ & $\begin{array}{c}\gamma \text { a } 28^{\circ} \mathrm{C} \\
\left(10^{-8} \mathrm{C} \mathrm{cm}^{-2} \mathrm{~K}^{-1}\right)\end{array}$ & $\begin{array}{c}\mathrm{F}_{\mathrm{V}} \\
\left(\mathrm{m}^{2} / \mathrm{C}\right)\end{array}$ & $\begin{array}{c}\mathrm{F}_{\mathrm{M}} \\
\left(10^{-6} \mathrm{~Pa}^{-1 / 2}\right)\end{array}$ \\
\hline $10 * 10 * 0.5 \mathrm{~mm}^{3}$ & 74 & 0.13 & 2.5 & 1.30 & 0.080 & 5.56 \\
\hline $10 * 10 * 0.2 \mathrm{~mm}^{3}$ & 150 & 0.07 & 2.5 & 1.74 & 0.053 & 7.20 \\
\hline
\end{tabular}

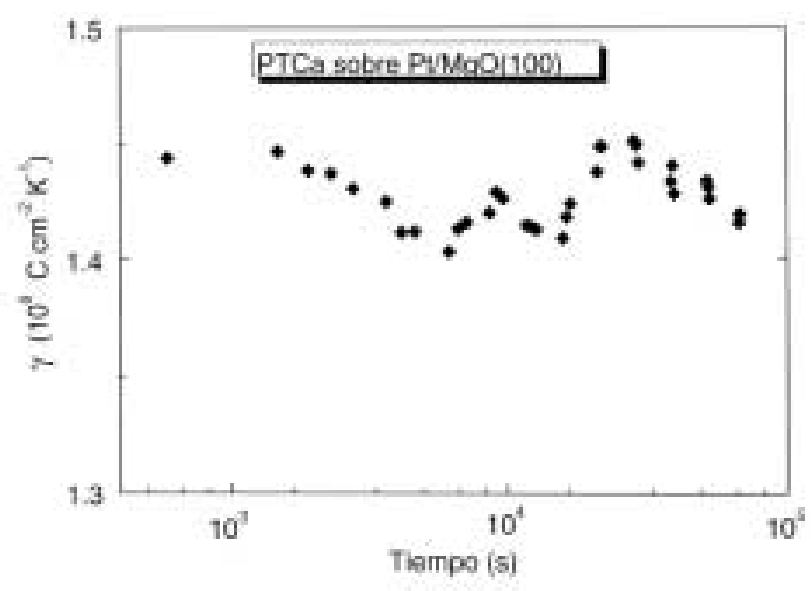

Figura 5. Evolución del coeficiente piroeléctrico con el tiempo de las láminas delgadas de $\left(\mathrm{Pb}_{0.76} \mathrm{Ca}_{0.24}\right) \mathrm{TiO}_{3}$ depositadas sobre $\mathrm{Pt} / \mathrm{MgO}(100)$ sin polarizar, no apreciándose degradación.

Así pues, para aumentar el valor de $\Delta \mathrm{T}$ hay que minimizar $G$ (aislamiento térmico) y maximizar $\eta$ (mediante el empleo de una capa absorbente). En el presente caso la manera de reducir $\mathrm{G}$, es disminuyendo el espesor de substrato, $\mathrm{L}$ que sustenta a los minicondensadores. Esto se consiguió después de un ataque químico controlado como se aprecia en el perfil de la parte inferior del substrato (figura 6). La reducción del espesor del substrato ha sido del 40\% (80 $\mu \mathrm{m}$ frente a un espesor inicial de $200 \mu \mathrm{m}$ ), que resultó insuficiente para ser eficaz en la reducción de pérdidas de calor por conducción. La reducción debe ser mucho mayor para obtener un $\Delta \mathrm{T}$ suficiente y conseguir una corriente eléctrica, I aceptable de 
acuerdo con:

$$
I=\gamma \mathrm{S} \frac{\mathrm{d} \Delta \mathrm{T}}{\mathrm{dt}}=\frac{\eta \gamma \mathrm{SP} \mathrm{o}_{\mathrm{o}} \omega}{\mathrm{G}\left(1+\omega^{2} \tau_{\mathrm{t}}^{2}\right)^{1 / 2}}
$$

El voltaje de salida $\left(\mathrm{V}_{\text {salida }}\right)$ dependerá de la admitancia total $Y$, según la ecuación 6 .

$$
V_{\text {salida }}=\frac{I}{Y}=\frac{\eta \gamma S P_{o} \omega R_{L} A}{G\left(1+\omega^{2} \tau_{t}^{2}\right)^{1 / 2}\left(1+\omega^{2} \tau_{e}^{2}\right)^{1 / 2}}
$$

donde $R_{L}$ es la resistencia de puerta (figura 3a), $S$ la superficie de los minicondensadores, A la ganancia de la electrónica y $\tau$. la constante de tiempo eléctrica, RC. La respuesta máxima se alcanzará para frecuencias de modulación entre $1 / \tau_{\mathrm{t}}$ y $1 / \tau_{\mathrm{e}^{\prime}}$

$$
\left(V_{\text {salida }}\right)_{\text {max }}=\frac{\eta \gamma P_{O} R_{L} S A}{G\left(\tau_{t}+\tau_{e}\right)}
$$

Puesto que la respuesta es inversamente proporcional a la conductancia térmica, parece razonable intentar minimizar su valor para aumentar la respuesta del detector. Del análisis de la conductancia térmica de la estructura de pozos del detector puede expresarse la conductancia mediante dos términos,

$$
\mathrm{G}=\mathrm{G}_{\mathrm{T}}+\mathrm{G}_{\text {Cond }}
$$

donde $\mathrm{G}_{\mathrm{T}}$ es la conductancia por emisión de radiación infrarroja que sigue la ley de Stephan-Boltzman, $\mathrm{G}_{\mathrm{T}}=4 \eta \sigma \mathrm{T}^{3} \mathrm{~S}$, siendo $\sigma$ la constante de Stephan-Boltzman y $\mathrm{G}_{\mathrm{Cond}}$ la conductancia por procesos de conducción térmica del elemento sensor a través del substrato. El segundo térmico puede dividirse a su vez en dos componentes: uno que corresponde a la conductancia térmica vertical $G_{V}$ (en la dirección de la radiación) y otro lateral $G_{L}$ calculada para dos dimensiones, ya que el proceso de conducción térmica sobre el substrato se produce en tres dimensiones.

En una primera aproximación al problema térmico [6] se obtiene la siguiente solución:

$$
\begin{array}{rlrl}
G_{\text {Cond }}=G_{V}+G & =k \frac{S}{L} \\
L & G_{L} & =k \frac{4 \pi L}{\ln \left(4 \pi L^{2} / S\right)}
\end{array}
$$

donde $\mathrm{k}$ es la conductividad térmica del substrato $\left(\sim 60 \mathrm{Wm}^{-1} \mathrm{~K}^{-}\right.$ 1). Del estudio de la dependencia de $G_{\text {Cond }}$ con $L$ se encuentra que es una relación de proporcionalidad, $\mathrm{G}_{\mathrm{Cond}} \propto \mathrm{kL}$. Tanto el efecto sobre la respuesta como sobre $\tau$ del detector son tales que para aumentarlas es necesario conseguir que el espesor del substrato sobre el que está el minicondensador sea mínimo.

$$
\begin{aligned}
\left(V_{\text {salida }}\right)_{\max } & \propto \frac{1}{G+H / \tau_{e}} \propto \frac{1}{k L+\left(G_{T}+H / \tau_{e}\right)} \\
\tau_{t} & =\frac{H}{G} \propto \frac{1}{G_{T}+k L}
\end{aligned}
$$

En la figura 7 se comprueba el comportamiento descrito, ya que se representa la respuesta del detector a diferentes frecuencias con diferentes espesores de substrato. Se observa que la respuesta disminuye al aumentar la frecuencia. También se aprecia que la respuesta del detector con un substrato de mayor espesor es considerablemente más baja. Está diferencia se hace más considerable a bajas frecuencias. Una figura de mérito de un detector piroeléctrico de IR es la detectividad especifica, D* que es la razón entre el voltaje de salida

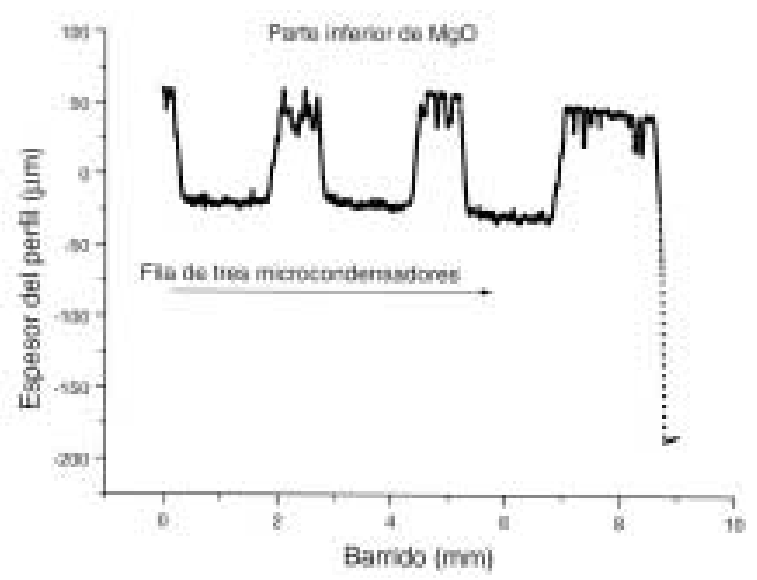

Figura 6. Perfil de la parte inferior del substrato de $\mathrm{MgO}$ después del ataque para formar pozos debajo de los minicondensadores.

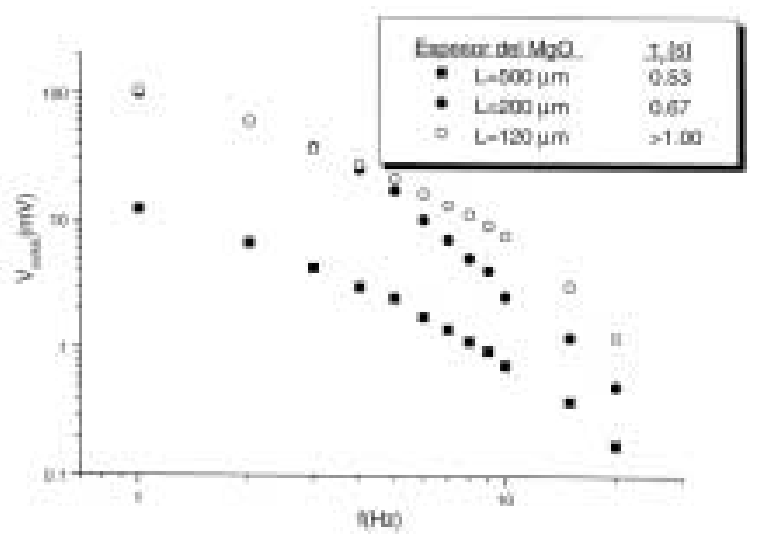

Figura 7. Voltaje de salida del detector frente a la frecuencia de modulación para diferente espesores de substratos.

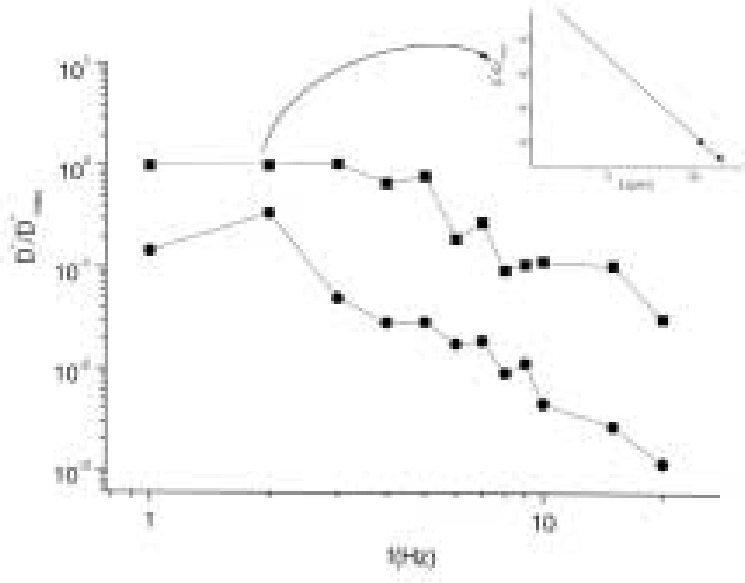

Figura 8. Detectividad especifica normalizada (al valor máximo alcanzado a $1 \mathrm{~Hz}$ para $\mathrm{L}=120 \mu \mathrm{m}$ ) frente a la frecuencia del detector piroeléctrico para dos espesores de $\mathrm{MgO}$ y su extrapolación para espesores menores.

y el de ruido para $1 \mathrm{~W}$ de potencia de radiación infrarroja detectada en $1 \mathrm{~cm}^{2}$ de área sensible [7]. El detector con un espesor de $\mathrm{MgO}$ de $\mathrm{L}=200 \mu \mathrm{m}$ muestra menos D" que después de reducir su espesor a $120 \mu \mathrm{m}$ (figura 8) debido a su alta conductancia térmica. Si este comportamiento se extrapolase empleando la ecuación 10 se obtendría que para espesores de $\mathrm{L} \sim 2 \mu \mathrm{m}$ la conductancia térmica se reduciría un factor 100, dando un aumento igual en D*. 


\section{CONCLUSIONES.}

Las láminas obtenidas por sol-gel sobre substratos de $\mathrm{MgO}(100)$ sometidos previamente a tratamientos térmicos presentan texturas cristalinas que dan lugar a coeficientes piroeléctricos espontáneos, incluso en minicondensadores de $7.10^{-3} \mathrm{~cm}^{2}$ de área. Debido a sus altos valores y a su escasa degradación temporal, estas láminas resultan muy útiles para la fabricación de detectores de IR (monoelemento o formadores de imágenes térmicas).

El aislamiento térmico del elemento sensor, para rentabilizar al máximo la conversión de radiación térmica en respuesta eléctrica, es el aspecto más importante que hay que cuidar en el diseño y construcción del detector. La reducción del espesor del substrato hasta espesores compatibles con la integridad del elemento sensor puede ser la vía más sencilla para la optimización de su respuesta.

\section{AGRADECIMIENTOS}

Este trabajo ha sido financiado por la CICYT a través del proyecto MAT98-1068. Los autores desean agradecerles a C. Alonso y M. Rus su valiosa ayuda en el diseño de la electrónica.

\section{BIBLIOGRAFÍA}

1. P. W. Kruse, D.D. Skatrud. "Uncooled Infrared Imaging Arrays and Systems". Semiconductors and Semimetals, Vol 47. Academic Press, 1997.

2. R. Jiménez, M.L. Calzada, J. Mendiola, "Preferred orientation of solutions derived $(\mathrm{Pb}, \mathrm{Ca}) \mathrm{TiO} 3$ thin films on $\mathrm{SrTiO}_{3}$ and $\mathrm{MgO}$ ", Proceeding of the $11^{\text {th }}$ IEEE International Symposium on applications of ferroelectrics, pp155-158. Ed. E. Colla, D. Damganovic and N. Setter. IEEE Catalog no 98-CH36245. Montreoux, Switzerland (1998).

3. R. Jimenez, P.Ramos, M.L. Calzada, J. Mendiola. “ Piroeléctricidad en láminas delgadas de titanato de plomo" Bol. Soc. Esp. Cerám. Vidrio, 37 (2-3), 117121 (1998)

4. A. González , R. Poyato, R. Jimenez, J. Mendiola, L. Pardo, M.L. Calzada, "Oriented growth of sol-gel modified PbTiO3 thin films on Si-based substrates", Surface Interface Anal., 29, 325-329 (2000).

5. S. Kim, S.Baik, "Deposition and characterizationf PT thin films grown by radio frequency" Thin Solid Films, 226, 205-211 (1995).

6. F. W. Schimdt, R.E. Henderson, C.H. Wolgemuth, " Introduction to thermal sciences" Pag. 315 Ed. Wiley (1993).

7. R.W. Whatmore, "Pyroelectric Devices and Materials", Rep. Prog. Phys. 49, 1335-1386 (1986)

Recibido: 31.05 .01

Aceptado: 10.12 .01 